\title{
Defining sets of full designs with block size three II
}

\author{
Diane Donovan, James Lefevre*广 Mary Waterhouse ${ }^{\ddagger}$ \\ Department of Mathematics, The University of Queensland \\ St Lucia Qld 4072, Australia \\ Emine Şule Yazıc1 ${ }^{\S}$ \\ Department of Mathematics, Koç University \\ 34450, Sarıyer, İstanbul, Turkey
}

AMS Subject Classification: 05B30

Keywords: defining sets, full designs

\begin{abstract}
A defining set of a $t-(v, k, \lambda)$ design is a subcollection of its blocks which is contained in a unique $t$-design with the given parameters. A minimal defining set is a defining set, none of whose proper subcollections is a defining set. The spectrum of minimal defining sets of a design $D$ is the set $\{|M| \mid M$ is a minimal defining set of $D\}$. The unique simple design with parameters $t-\left(v, k,\left(\begin{array}{l}v-t \\ k-t\end{array}\right)\right)$ is said to be the full design on $v$ elements. This paper studies the minimal defining sets of full designs when $t=2$ and $k=3$. The largest known minimal defining set is given. The existence of a continuous section of the spectrum comprising asymptotically $9 v^{2} / 50$ values is shown. This gives a quadratic length section of continuous spectrum where only a linear section with respect to $v$ was known before.
\end{abstract}

*Donovan and Lefevre supported by grants DP0664030 and LX0453416

†Current e-mail:James.Lefevre@sami.org.au

${ }^{\ddagger}$ Current e-mail: mwaterhouse@wesleyresearch.com.au

$\S$ This work was carried out at The University of Queensland and Yazıcı was supported by Raybould fellowship and TUBITAK CAREER grant 106T574 


\section{Introduction}

A block design is a pair $(V, \mathcal{B})$, where $V$ is a $v$-set of elements and $\mathcal{B}$ is a collection of $b$ $k$-subsets (called blocks) chosen from $V$ such that every element of $V$ occurs in exactly $r$ blocks. We refer to $v$ and $b$ as the order and size of the design respectively. If every $t$-subset of $V$ belongs to exactly $\lambda$ blocks, then we say that the design is $t$-balanced, and we call the block design a $t$-design with parameters $t-(v, k, \lambda)$. When $t=2$ we refer to this more simply as a $(v, k, \lambda)$ design.

A design is said to be simple if it doesn't contain any repeated blocks. The unique simple balanced design with parameters $\left(v, k,\left(\begin{array}{l}v-2 \\ k-2\end{array}\right)\right)$ is said to be the full design on $v$ elements. It comprises all possible $k$-tuples on the $v$-set $V$, and is therefore the largest possible simple design on $v$ elements.

A set of the blocks which is a subset of an unique $(v, k, \lambda)$ design is said to be a defining set of the design, denoted $d(v, k, \lambda)$. A defining set is said to be minimal if none of its proper subsets is a defining set, denoted $d_{m}(v, k, \lambda)$. A defining set is said to be smallest if no other defining set of the $(v, k, \lambda)$ design has smaller cardinality, denoted $d_{s}(v, k, \lambda)$. The spectrum of a design $D$ is defined $\operatorname{spec}(D)=\{|M| \mid M$ is a minimal defining set of $D\}$. A continuous portion of the spectrum comprising $n$ values is a set of $n$ consecutive positive integers where for each element of the set there exists at least one minimal defining set with that size.

Let $(V, \mathcal{B})$ be a $(v, k, \lambda)$ design and suppose that $X \subseteq \mathcal{B}$. Then any $(v, k, \lambda)$ design on underlying set $V$ which contains the blocks of $X$ is a completion of $X$. Any block which is not in $X$ but is in every completion of $X$ is said to be forced (by $X$ ). Thus a defining set is a subdesign which has a unique completion, or equivalently one for which every block which is in the design but not the subdesign is forced.

Defining sets of $t$-designs were first introduced by Ken Gray. He gave preliminary results and lower bounds for the smallest defining sets of some families of these designs in a series of papers $[5,6,7]$. Some theoretical techniques and many different algorithms were suggested for finding smallest and minimal defining sets of combinatorial designs but the minimal defining sets of only a limited number of designs were classified previously $[3,4,11,13]$. See [10] and [12] for an overview of recent improvements.

In [2] it is shown that every minimal defining set (including each of the smallest ones) of a simple $(v, k, \lambda)$ design is the intersection of the design with a minimal defining set of the $\left(v, k,\left(\begin{array}{l}v-2 \\ k-2\end{array}\right)\right)$ full design. So using the classifications of minimal defining sets of full designs is a comprehensive method for determining the spectrum of all $(v, k, \lambda)$ designs. Also in [8] it was shown that the sizes of the minimal defining sets of full designs with $v$ elements can be 
used to find upper bounds for the number of designs with parameters $(v, k, \lambda)$.

We are interested in minimal defining sets of full designs with block size three. From here onwards, we use full design to refer to the full design with block size three, and block will denote a 3 -subset. Let $F(V)(=\{\{x, y, z\} \mid x, y, z \in V, x, y, z$ distinct $\})$ denote the full design on the set $V$ with parameters $(v, 3, v-2)$. If $D$ is a defining set of $F(V)$, then $D^{C}=F(V) \backslash D$.

The term $d(v)$ will denote a defining set of the full design on some $v$-set. The term $d_{m}(v)$ will denote a minimal $d(v)$. We are interested in determining the spectrum of allowed sizes for a $d_{m}(v)$ of $F(V)$, for each $v=|V|$.

A trade is a set of blocks $T$ for which there exists a second entirely disjoint set of blocks $T^{\prime}$, with the property that every pair of elements in the underlying set occurs equally often in blocks of $T$ and in blocks of $T^{\prime}$. We refer to $T^{\prime}$ as the disjoint mate of $T$. Trades are related to minimal defining sets through the following well-known result, first given in [5]:

Lemma 1.1 Let $D$ be a subset of the blocks of a design $(V, \mathcal{B})$, and let $D^{C}=\mathcal{B} \backslash D$. Then

(1) $D$ is a defining set of $(V, \mathcal{B})$ if, and only if, there is no trade $T \subseteq D^{C}$; that is, every trade contained in $\mathcal{B}$ intersects $D$.

(2) Suppose that $D$ is a defining set of $(V, \mathcal{B})$. Then $D$ is minimal if, and only if, for every block $B \in D$ there exists a trade $T$ satisfying $T \backslash D^{C}=\{B\}$.

The smallest possible trade is known as the Pasch trade. For distinct elements $u, v$, $w, x, y, z$, the Pasch trade has $T=\{\{u, w, x\},\{u, y, z\},\{v, w, y\},\{v, x, z\}\}$ and $T^{\prime}=$ $\{\{v, w, x\},\{v, y, z\},\{u, w, y\},\{u, x, z\}\}$. Another trade of which we will make frequent use is given by $T=\{\{t, w, x\},\{t, v, y\},\{t, v, z\},\{u, y, z\},\{u, v, w\},\{u, v, x\}\}$, where $t, u, v, w$, $x, y, z$ are distinct elements. Its disjoint mate is given by $T^{\prime}=\{\{u, w, x\},\{u, v, y\},\{u, v, z\}$, $\{t, y, z\},\{t, v, w\},\{t, v, x\}\}$. We will refer to a trade with this structure as a Type 2 trade.

In [1] and [13] constructions are given which produce $d_{m}(v)$ s of size $\left(v^{3}-6 v^{2}+5 v+12\right) / 6$, $v \geq 6$, and $\left(v^{3}-6 v^{2}+11 v-30\right) / 6, v \geq 5$, respectively. These are listed as Constructions 1 and 2 in [2]. A more general form of Construction 2 for block sizes greater than three is given in [9]. In [2] the authors gave more general constructions and developed bounds on the size of $d_{m}(v) \mathrm{s}$, as summarized by the following lemma. 
Lemma 1.2 Let $v \geq 7$.

(1) There exists a $d_{m}(v)$ of size $n$ if $\frac{v^{3}-6 v^{2}+5 v+6}{6} \leq n \leq \frac{v^{3}-6 v^{2}+11 v-24}{6}$.

(2) If there exists a $d_{m}(v)$ of size $n$, then $\frac{15}{35}\left(\begin{array}{l}v \\ 3\end{array}\right) \leq n \leq\left(\begin{array}{l}v \\ 3\end{array}\right)-v$.

See [2] and [8] for further background and motivation for our study of the defining sets of the full design.

Asymptotically, the established spectrum contains approximately $v$ consecutive values. However, the gap between the highest known value and the upper bound is of order $v^{2}$, while the gap between the smallest known value and the lower bound is of order $v^{3}$.

We improve the known spectrum; our main results are given in Theorems 3.1 and 3.2. The higher end of the spectrum of known values is increased to include, asymptotically, $9 v^{2} / 50$ consecutive values. The difference between the size of the largest known $d_{m}(v)$ and the upper bound is reduced to $2 v-5$ by Lemma 2.2 with $k=1$.

In Section 2 we give our new constructions, and in Section 3 we calculate the values of the spectrum covered by our constructions.

\section{Constructions}

We develop some of our constructions with the aid of the following well-known lemma.

Lemma 2.1 Let $U$ and $W$ be disjoint sets of size $u$ and $w$ respectively. Let $D$ be a $d_{m}(u)$ of $F(U)$, and let $D^{*}$ be a set of blocks on $U \cup W$ such that $D^{*} \cap F(U)=D$. Then

(1) $D^{*}$ is a $d(u+w)$ of $F(U \cup W)$ if, and only if, all blocks in $\left(D^{*}\right)^{C} \backslash D^{C}$ are forced by $D^{*}$; and

(2) $D^{*}$ is a $d_{m}(u+w)$ of $F(U \cup W)$ if, and only if, it is a $d(u+w)$ and for every block $B \in D^{*} \backslash D$ there exists a trade $T$ with $T \backslash\left(D^{*}\right)^{C}=\{B\}$.

The following minimal defining set obtained by Construction 1 is the main tool we will use to obtain the results in this paper. We will extend these minimal defining sets by using the extension constructions, Construction 2 and 3 to get minimal defining sets with consecutive sizes for each $v$. 
Construction 1 Let $\{0,1\}, U_{1}, U_{2}, \ldots, U_{k}$ and $S$ be disjoint sets with $U_{i}=\left\{x_{i}, y_{i}, z_{i}\right\}$, $1 \leq i \leq k$, and $|S|=s \geq 0$. Let $V=\{0,1\} \cup \bigcup_{i=1}^{k} U_{i} \cup S$, and $v=|V|=3 k+2+s$. Define

$$
D_{1}=F(V) \backslash\left(\bigcup_{i=1}^{k} F\left(\{0,1\} \cup U_{i}\right) \cup \bigcup_{\alpha \in S}\left\{\{0,1, \alpha\},\left\{0, x_{1}, \alpha\right\},\left\{1, x_{1}, \alpha\right\}\right\}\right) .
$$

Lemma 2.2 The set $D_{1}$ defined by Construction 1 is a $d_{m}(v)$ of $F(V)$ of size $\left(v^{3}-3 v^{2}-\right.$ $16 v+36) / 6-k$.

Proof: The cardinality of $D_{1}$ is given by $\left(\begin{array}{l}v \\ 3\end{array}\right)-k\left(\begin{array}{l}5 \\ 3\end{array}\right)-3 s$. We consider two cases.

Case 1. $s=0$ : To show unique completion, let $C=D_{1} \cup R$ be a completion of $D_{1}$ to a $(v, 3, v-2)$ design on $V$; we will show that $C=F(V)$. If $x \in U_{i}$ and $y \in U_{j}, i \neq j$, then there is no block in $R$ containing $\{x, y\}$. Thus every block in $R$ is a subset of some $U_{i} \cup\{0,1\}$.

Let $R_{i}$ denote the subset of $R$ comprising blocks in $U_{i} \cup\{0,1\}$. For any pair contained in $U_{i} \cup\{0,1\}$, other than $\{0,1\}$, there are exactly three blocks in $R_{i}$ containing that pair. Thus if $\{0,1\}$ occurs in $n$ blocks of $R_{i}$, then $\left|R_{i}\right|=\frac{9 \cdot 3+n}{3}=9+\frac{n}{3}$.

Suppose that there is some $j \in\{1,2, \ldots, k\}$ such that $\left|R_{j}\right| \neq 10$. Since $\sum_{i=1}^{k}\left|R_{i}\right|=|R|$ $=\left|D_{1}^{C}\right|=10 k$, we can assume without loss of generality that $\left|R_{j}\right|<10$; it follows that $\left|R_{j}\right|=9$ and $\{0,1\}$ occurs in no blocks of $R_{j}$. However, for each $x \in U_{j},\{0, x\}$ occurs in three blocks of $R_{j}$, implying that there is a total of $9 / 2$ blocks in $R_{j}$ which contain 0 , a contradiction. Therefore $\left|R_{i}\right|=10$, for $i=1,2, \ldots, k$, and each $R_{i}$ forms a $(5,3,3)$ design on $U_{i} \cup\{0,1\}$. Since the unique $(5,3,3)$ design is the full design of order five, $R=D_{1}^{C}$ as required.

To show minimality we need to show that for every $X \in D_{1}$ there exists a trade $T$ satisfying $T \backslash D_{1}^{C}=\{X\}$. Up to generality, we can assume that $X$ is of the form $\left\{0, x_{1}, x_{2}\right\}$, $\left\{x_{1}, y_{1}, x_{2}\right\}$ or $\left\{x_{1}, x_{2}, x_{3}\right\}$.

For $X=\left\{0, x_{1}, x_{2}\right\}$, an appropriate trade is $\left\{\left\{0, x_{1}, x_{2}\right\},\left\{0,1, x_{2}\right\},\left\{0,1, y_{2}\right\},\left\{0,1, z_{2}\right\}\right.$, $\left.\left\{0, y_{1}, z_{1}\right\},\left\{x_{2}, y_{2}, z_{2}\right\},\left\{1, x_{1}, y_{1}\right\},\left\{1, x_{1}, z_{1}\right\}\right\}$, with disjoint mate $\left\{\left\{1, x_{1}, x_{2}\right\},\left\{0,1, x_{1}\right\},\{0,1\right.$, $\left.\left.y_{1}\right\},\left\{0,1, z_{1}\right\},\left\{1, y_{2}, z_{2}\right\},\left\{x_{1}, y_{1}, z_{1}\right\},\left\{0, x_{2}, y_{2}\right\},\left\{0, x_{2}, z_{2}\right\}\right\}$. For $X=\left\{x_{1}, y_{1}, x_{2}\right\}$, a suitable (Pasch) trade is: $\left\{\left\{x_{1}, y_{1}, x_{2}\right\},\left\{x_{1}, z_{1}, 0\right\},\left\{1, y_{1}, z_{1}\right\},\left\{1, x_{2}, 0\right\}\right\}$. For $X=\left\{x_{1}, x_{2}, x_{3}\right\}$, the required (Type 2) trade is $\left\{\left\{x_{1}, x_{2}, x_{3}\right\},\left\{x_{1}, 0, y_{1}\right\},\left\{x_{1}, 0, z_{1}\right\},\left\{1, y_{1}, z_{1}\right\},\left\{1,0, x_{2}\right\},\left\{1,0, x_{3}\right\}\right\}$.

Case 2. $s>0$ : Let $U=\{0,1\} \cup \bigcup_{i=1}^{k} U_{i}$. Note that $D_{1} \cap F(U)$ is the $d_{m}(3 k+2)$ of $F(U)$ given by Case 1 . Thus, by Lemmas 2.1 and Case 1, when proving unique completion and 
minimality, it is only necessary to consider blocks containing at least one element of $S$.

To show unique completion, let $C=D_{1} \cup R$ be a completion of $D_{1}$ to a $(v, 3, v-2)$ design on $V$. Let $\alpha$ be an arbitrary element of $S$. The element $\alpha$ occurs with each of the elements 0,1 and $x_{1}$ in exactly two blocks of $D_{1}^{C}$ and hence $R$. Furthermore, $\alpha$ does not occur with any other elements in blocks of $R$. It follows immediately that $R$ must contain $\{0,1, \alpha\},\left\{0, x_{1}, \alpha\right\}$ and $\left\{1, x_{1}, \alpha\right\}$. Thus $R=D_{1}^{C}$ as required.

Up to loss of generality, to show minimality we need to find a trade $T$ satisfying $T \backslash$ $D_{1}^{C}=\{X\}$ for $X$ of the form $\left\{\alpha_{1}, 0, y_{1}\right\},\left\{\alpha_{1}, 0, x_{2}\right\},\left\{\alpha_{1}, x_{1}, y_{1}\right\},\left\{\alpha_{1}, x_{1}, x_{2}\right\},\left\{\alpha_{1}, y_{1}, z_{1}\right\}$, $\left\{\alpha_{1}, y_{1}, x_{2}\right\},\left\{\alpha_{1}, x_{2}, y_{2}\right\},\left\{\alpha_{1}, x_{2}, x_{3}\right\},\left\{\alpha_{1}, \alpha_{2}, 0\right\},\left\{\alpha_{1}, \alpha_{2}, x_{1}\right\},\left\{\alpha_{1}, \alpha_{2}, y_{1}\right\},\left\{\alpha_{1}, \alpha_{2}, x_{2}\right\}$ or $\left\{\alpha_{1}, \alpha_{2}, \alpha_{3}\right\}$, where $\alpha_{1}, \alpha_{2}$ and $\alpha_{3}$ are three distinct points in $S$; here $\alpha_{2}$ and $\alpha_{3}$ are only used in the cases where $|S| \geq 2$ and $|S| \geq 3$, respectively.

For $X \in\left\{\left\{\alpha_{1}, 0, y_{1}\right\},\left\{\alpha_{1}, x_{1}, y_{1}\right\},\left\{\alpha_{1}, y_{1}, z_{1}\right\},\left\{\alpha_{1}, y_{1}, x_{2}\right\},\left\{\alpha_{1}, x_{2}, y_{2}\right\},\left\{\alpha_{1}, \alpha_{2}, y_{1}\right\},\left\{\alpha_{1}\right.\right.$, $\left.\left.\alpha_{2}, x_{2}\right\},\left\{\alpha_{1}, \alpha_{2}, \alpha_{3}\right\}\right\}$ suitable (Pasch) trades are:

$$
\begin{array}{lll}
\left\{\left\{\alpha_{1}, 0, y_{1}\right\},\left\{x_{1}, y_{1}, z_{1}\right\},\left\{x_{1}, \alpha_{1}, 1\right\},\left\{0, z_{1}, 1\right\}\right\}, & \left\{\left\{\alpha_{1}, x_{2}, y_{2}\right\},\left\{\alpha_{1}, 0,1\right\},\left\{z_{2}, 0, x_{2}\right\},\left\{z_{2}, 1, y_{2}\right\}\right\}, \\
\left\{\left\{\alpha_{1}, x_{1}, y_{1}\right\},\left\{0, y_{1}, z_{1}\right\},\left\{0, \alpha_{1}, 1\right\},\left\{x_{1}, z_{1}, 1\right\}\right\}, & \left\{\left\{\alpha_{1}, \alpha_{2}, y_{1}\right\},\left\{1, x_{1}, y_{1}\right\},\left\{0,1, \alpha_{1}\right\},\left\{0, x_{1}, \alpha_{2}\right\}\right\}, \\
\left\{\left\{\alpha_{1}, y_{1}, z_{1}\right\},\left\{0,1, z_{1}\right\},\left\{0, x_{1}, y_{1}\right\},\left\{1, x_{1}, \alpha_{1}\right\}\right\}, & \left\{\left\{\alpha_{1}, \alpha_{2}, x_{2}\right\},\left\{0,1, x_{2}\right\},\left\{0, \alpha_{1}, x_{1}\right\},\left\{1, \alpha_{2}, x_{1}\right\}\right\} \\
\left\{\left\{\alpha_{1}, y_{1}, x_{2}\right\},\left\{\alpha_{1}, 1, x_{1}\right\},\left\{0,1, x_{2}\right\},\left\{0, x_{1}, y_{1}\right\}\right\}, & \left\{\left\{\alpha_{1}, \alpha_{2}, \alpha_{3}\right\}\left\{\alpha_{1}, 0,1\right\},\left\{\alpha_{2}, 0, x_{1}\right\},\left\{\alpha_{3}, 1, x_{1}\right\}\right\} .
\end{array}
$$

For $X \in\left\{\left\{\alpha_{1}, x_{1}, x_{2}\right\},\left\{\alpha_{1}, x_{2}, x_{3}\right\},\left\{\alpha_{1}, \alpha_{2}, 0\right\},\left\{\alpha_{1}, \alpha_{2}, x_{1}\right\}\right\}$ suitable (Type 2) trades are:

$$
\begin{aligned}
& \left\{\left\{x_{2}, \alpha_{1}, x_{1}\right\},\left\{x_{2}, 0, y_{2}\right\},\left\{x_{2}, 0, z_{2}\right\},\left\{1, y_{2}, z_{2}\right\},\left\{1,0, \alpha_{1}\right\},\left\{1,0, x_{1}\right\}\right\}, \\
& \left\{\left\{x_{2}, \alpha_{1}, x_{3}\right\},\left\{x_{2}, 1, y_{2}\right\},\left\{x_{2}, 1, z_{2}\right\},\left\{0, y_{2}, z_{2}\right\},\left\{0,1, \alpha_{1}\right\},\left\{0,1, x_{3}\right\}\right\}, \\
& \left\{\left\{0, \alpha_{1}, \alpha_{2}\right\},\left\{0,1, y_{1}\right\},\left\{0,1, z_{1}\right\},\left\{x_{1}, y_{1}, z_{1}\right\},\left\{x_{1}, 1, \alpha_{1}\right\},\left\{x_{1}, 1, \alpha_{2}\right\}\right\} \text { and } \\
& \left\{\left\{x_{1}, \alpha_{1}, \alpha_{2}\right\},\left\{x_{1}, 1, y_{1}\right\},\left\{x_{1}, 1, z_{1}\right\},\left\{0, y_{1}, z_{1}\right\},\left\{0,1, \alpha_{1}\right\},\left\{0,1, \alpha_{2}\right\}\right\} .
\end{aligned}
$$

For $X=\left\{\alpha_{1}, 0, x_{2}\right\}$ the required trade is $\left\{\left\{\alpha_{1}, 0, x_{2}\right\},\left\{0,1, x_{2}\right\},\left\{0,1, y_{2}\right\},\left\{0,1, z_{2}\right\},\left\{0, x_{1}\right.\right.$, $\left.\left.y_{1}\right\},\left\{0, x_{1}, z_{1}\right\},\left\{1, x_{1}, \alpha_{1}\right\},\left\{1, y_{1}, z_{1}\right\},\left\{x_{2}, y_{2}, z_{2}\right\}\right\}$, with disjoint mate $\left\{\left\{\alpha_{1}, 0, x_{1}\right\},\left\{0,1, x_{1}\right\}\right.$, $\left.\left\{0,1, y_{1}\right\},\left\{0,1, z_{1}\right\},\left\{0, x_{2}, y_{2}\right\},\left\{0, x_{2}, z_{2}\right\},\left\{1, x_{2}, \alpha_{1}\right\},\left\{1, y_{2}, z_{2}\right\},\left\{x_{1}, y_{1}, z_{1}\right\}\right\}$.

The following results allow us to extend Construction 1.

Lemma 2.3 [2] Let $U$ and $W$ be disjoint sets of size $u \geq 5$ and $w \geq 0$ respectively, let $D$ be $a d_{m}(u)$ of $F(U)$ of size $n$, and let $a \in U$. Let $V=U \cup W$ and $v=|V|$. Define $D^{*}=D \cup(F(V \backslash\{a\}) \backslash F(U))$. Then the set $D^{*}$ is:

(1) a d (v) of $F(V)$ of size $n+\left(\begin{array}{c}v-1 \\ 3\end{array}\right)-\left(\begin{array}{c}u-1 \\ 3\end{array}\right)$; and

(2) minimal if the following condition holds: for every $x_{1}, x_{2} \in U \backslash\{a\}$, and for an arbitrary element $p \notin U$, there exists a trade $T$ with $T \backslash\left(D^{C} \cup\{\{p, a, x\} \mid x \in U \backslash\{a\}\}\right)=$ $\left\{\left\{p, x_{1}, x_{2}\right\}\right\}$. 
Lemma 2.4 [2] Let $U, W$ and $Q$ be disjoint sets of size $u \geq 5, w \geq 2$ and $q \geq 1$, respectively, and let $V=U \cup W \cup Q$ and $v=|V|$. Let $a, b \in U$ and $c \in Q$. Assume that $D$ is $a d_{m}(u)$ of $F(U)$ of size $n$, and let $D^{C}=F(U) \backslash D$. Define

$$
\begin{aligned}
\left(D^{*}\right)^{C}= & D^{C} \cup\left\{\left\{a, y_{1}, x\right\},\left\{a, y_{1}, y_{2}\right\},\left\{a, y_{1}, c\right\} \mid x \in U \backslash\{a\}, y_{1}, y_{2} \in W\right\} \\
& \cup\left\{\left\{b, z_{1}, x\right\},\left\{b, z_{1}, z_{2}\right\},\left\{b, z_{1}, y\right\} \mid x \in U \backslash\{b\}, z_{1}, z_{2} \in Q, y \in W\right\} .
\end{aligned}
$$

Then $\left(D^{*}\right)^{C} \subseteq F(V)$ and $D^{*}=F(V) \backslash\left(D^{*}\right)^{C}$ is

(1) a d (v) of $F(V)$ of size $n+\left(\begin{array}{c}v-1 \\ 3\end{array}\right)-\left(\begin{array}{c}u-1 \\ 3\end{array}\right)-w$; and

(2) minimal if for every $x_{1}, x_{2} \in U \backslash\{\alpha\}$, where $\alpha \in\{a, b\}$, and for an arbitrary element $p \notin U$, there exists a trade $T$ with $T \backslash\left(D^{C} \cup\{\{p, \alpha, x\} \mid x \in U \backslash\{\alpha\}\}\right)=\left\{\left\{p, x_{1}, x_{2}\right\}\right\}$.

Construction 2 Let $\{0,1\}, U_{1}, U_{2}, \ldots, U_{k}, S$ and $W$ be disjoint sets with $\left|U_{i}\right|=\left\{x_{i}, y_{i}, z_{i}\right\}$, $1 \leq i \leq k,|S|=s \geq 0$, and $|W|=w \geq 0$. Define $U=\{0,1\} \cup \bigcup_{i=1}^{k} U_{i} \cup S, u=|U|=$ $3 k+2+s, V=U \cup W$, and $v=|V|=u+w$. Note that $v \geq 5$.

Let $D_{1}$ be as defined in Construction 1 , but on underlying set $U$ rather than $V$. Define

$$
D_{2}=D_{1} \cup(F(V \backslash\{0\}) \backslash F(U)) .
$$

Construction 3 Let $\{0,1\}, U_{1}, U_{2}, \ldots, U_{k}, S, W$ and $Q$ be disjoint sets with $\left|U_{i}\right|=$ $\left\{x_{i}, y_{i}, z_{i}\right\}, 1 \leq i \leq k,|S|=s \geq 0,|W|=w^{\prime} \geq 2$ and $\underset{k}{|Q|}=q \geq 1$. Let $c \in Q$ be a distinguished point, and let $w=w^{\prime}+q$. Define $U=\{0,1\} \cup \bigcup_{i=1}^{k} U_{i} \cup S, u=|U|=3 k+2+s$, $V=U \cup W \cup Q$, and $v=|V|=u+w$. Note that $v \geq 8$.

Let $D_{1}$ be as defined in Construction 1 , but on underlying set $U$ rather than $V$. Define

$$
\begin{aligned}
D_{3} & =D_{1} \cup\left(F ( V ) \backslash \left(F(U) \cup\left\{\left\{0, \beta_{1}, \alpha\right\},\left\{0, \beta_{1}, \beta_{2}\right\},\left\{0, \beta_{1}, c\right\} \mid \alpha \in U \backslash\{0\}, \beta_{1}, \beta_{2} \in W\right\}\right.\right. \\
& \left.\left.\cup\left\{\left\{1, \gamma_{1}, \alpha\right\},\left\{1, \gamma_{1}, \gamma_{2}\right\},\left\{1, \gamma_{1}, \beta\right\} \mid \alpha \in U \backslash\{1\}, \gamma_{1}, \gamma_{2} \in Q, \beta \in W\right\}\right)\right) .
\end{aligned}
$$

Lemma 2.5 The sets $D_{2}$ and $D_{3}$ defined by Constructions 2 and 3 are $d_{m}(v) s$ of sizes

$$
\begin{aligned}
& \frac{v^{3}-3 v^{2}-16 v+36}{6}-\frac{w(2 v-9-w)}{2}-k \text { and } \\
& \frac{v^{3}-3 v^{2}-16 v+36}{6}-\frac{w(2 v-9-w)}{2}-w^{\prime}-k, \text { respectively. }
\end{aligned}
$$




\section{Proof.}

By Lemma 2.2, the set $D_{1}$ used in Constructions 2 and 3 is a $d_{m}(u)$ of size $\left(u^{3}-3 u^{2}-\right.$ $16 u+36) / 6-k$. The sizes of $D_{2}$ and $D_{3}$ follow by Lemmas 2.3 and 2.4 respectively (or by direct count). By Lemma 2.3 (with $D, D^{*}$ and $a$ corresponding to $D_{1}, D_{2}$ and 0 respectively), to prove that $D_{2}$ is a $d_{m}(v)$ it is only necessary to find, for an arbitrary $p \notin U$ and for every $\alpha_{1}, \alpha_{2} \in U \backslash\{0\}$, a trade $T$ satisfying $T \backslash\left(D_{1}^{C} \cup\{\{p, 0, \alpha\} \mid \alpha \in U \backslash\{0\}\}\right)=\left\{\left\{p, \alpha_{1}, \alpha_{2}\right\}\right\}$. We give these trades below. By symmetry, we can substitute 1 for 0 in this condition; thus by Lemma 2.4 (with $D, D^{*} a$ and $b$ corresponding to $D_{1}, D_{3}, 0$ and 1 respectively), it follows that $D_{3}$ is also a $d_{m}(v)$.

We begin by considering the case where neither point is in $S$; that is, $\alpha_{1}, \alpha_{2} \in\{1\} \cup$ $\bigcup_{i=1}^{k} U_{i}$. Since the following trades do not use any elements of $S$, we can regard $U_{i}$ and $U_{j}$ as interchangable, and $x_{i}, y_{i}, z_{i}$ as interchangable, for every $i$ and $j$. Thus up to generality we have $\left\{\alpha_{1}, \alpha_{2}\right\} \in\left\{\left\{1, x_{1}\right\},\left\{x_{1}, y_{1}\right\},\left\{x_{1}, x_{2}\right\}\right\}$. Suitable (Pasch) trades are $\left\{\left\{p, 1, x_{1}\right\},\left\{p, 0, y_{1}\right\},\left\{0,1, z_{1}\right\},\left\{x_{1}, y_{1}, z_{1}\right\}\right\},\left\{\left\{p, x_{1}, y_{1}\right\},\{p, 0,1\},\left\{0, x_{1}, z_{1}\right\},\left\{1, y_{1}, z_{1}\right\}\right\}$ and $\left\{\left\{p, x_{1}, x_{2}\right\},\left\{p, 0, y_{1}\right\},\left\{1,0, x_{2}\right\},\left\{1, x_{1}, y_{1}\right\}\right\}$.

If $\alpha_{1}, \alpha_{2} \in S$, the required (Pasch) trade is: $\left\{\left\{p, \alpha_{1}, \alpha_{2}\right\},\{p, 0,1\},\left\{\alpha_{1}, 0, x_{1}\right\},\left\{\alpha_{2}, 1, x_{1}\right\}\right\}$.

We are left with the case $\alpha_{1} \in S, \alpha_{2} \notin S$. Up to generality we have $\alpha_{2}$ equal to 1 , $x_{1}, y_{1}$ or $x_{2}$. The required (Pasch) trades are $\left\{\left\{p, \alpha_{1}, 1\right\},\left\{p, 0, y_{1}\right\},\left\{0, x_{1}, \alpha_{1}\right\},\left\{1, x_{1}, y_{1}\right\}\right\}$, $\left\{\left\{p, \alpha_{1}, x_{1}\right\},\left\{p, 0, y_{1}\right\},\left\{0,1, \alpha_{1}\right\},\left\{1, x_{1}, y_{1}\right\}\right\},\left\{\left\{p, \alpha_{1}, y_{1}\right\},\left\{p, 0, x_{1}\right\},\left\{0,1, \alpha_{1}\right\},\left\{1, x_{1}, y_{1}\right\}\right\}$ and $\left\{\left\{p, \alpha_{1}, x_{2}\right\},\left\{p, 0, y_{2}\right\},\left\{0,1, \alpha_{1}\right\},\left\{1, x_{2}, y_{2}\right\}\right\}$.

\section{Evaluating the spectrum}

We now calculate, for a given $v$, the range of $d_{m}(v)$ sizes given by Constructions 2 and 3 .

Theorem 3.1 For any integers $v, w$ satisfying $0 \leq w \leq v-5$, let

$$
\Omega_{v, w}=\frac{v^{3}-3 v^{2}-16 v+30}{6}-\frac{w(2 v-9-w)}{2} \text { and } \Delta_{v, w}=\left\lfloor\frac{v+2 w-8}{3}\right\rfloor .
$$

Then there exists a $d_{m}(v)$ of size $n$ provided that $\Omega_{v, w}-\Delta_{v, w} \leq n \leq \Omega_{v, w}$, except that if $w \geq v-7$ then there may not be a $d_{m}(v)$ of size $\Omega_{v, w}-1$. In addition, there is a $d_{m}(v)$ of size $\Omega_{v, 0}-\left\lfloor\frac{v-5}{3}\right\rfloor=\Omega_{v, 0}-\Delta_{v, 0}-1$.

Proof. In Constructions 2 and 3, we have $v=3 k+2+s+w$, where $k \geq 1, s \geq 0$ and $w \geq 0$, and in Construction 3 we also have $2 \leq w^{\prime} \leq w-1$ (implying that $w \geq 3$ ). Thus 
for a fixed $v$ and $w$, which must satisfy $0 \leq w \leq v-5, k$ and $w^{\prime}$ can take any integer value satisfying $1 \leq k \leq(v-w-2) / 3$ and $2 \leq w^{\prime} \leq w-1$. The result follows from Lemma 2.5.

Note that Construction 2 gives a defining set of size equivalent to the defining set given by Construction 3 with $w^{\prime}=0$. The fact that $w^{\prime} \neq 1$ only matters when there is no flexibility in choosing $k$; that is, the upper bound on $k$ is less than 2 , or equivalently $w \geq v-7$. In this case neither construction can be of size $\Omega_{v, w}-1$.

Theorem 3.2 Let $v \geq 12$, and let $r \equiv-2 v(\bmod 5)$, with $0 \leq r<5$. Then there exists a $d_{m}(v)$ of size $n$ provided that

$$
\frac{v^{3}-6 v^{2}+5 v+6}{6} \leq n \leq \frac{25 v^{3}-123 v^{2}+v(50-18 r)+\left(3 r^{2}+75 r-300\right)}{150} .
$$

Proof. Let $w$ take any integer value in the range $0 \leq w \leq v-5$, and let $\Omega_{v, w}$ and $\Delta_{v, w}$ be as defined in Theorem 3.1. Note that $\Omega_{v, w}$ is a decreasing function of $w$. Consider the range of $d_{m}(v)$ sizes given by Theorem 3.1 for each $w$. The smallest size when $w=m$ (namely $\Omega_{v, m}-\Delta_{v, m}$ ) is at most one more than the largest size when $w=m+1$ (namely $\Omega_{v, m+1}$ ) if and only if $-\frac{m(2 v-9-m)}{2}-\left\lfloor\frac{v+2 m-8}{3}\right\rfloor \leq-\frac{(m+1)(2 v-9-(m+1))}{2}+1$; that is, $m \geq w_{0}$, where $w_{0}=$ $\left\lceil\frac{2 v-10}{5}\right\rceil$. Thus, by Theorem 3.1, we have a $d_{m}(v)$ of every size between $\Omega_{v, v-5}-\Delta_{v, v-5}$ and $\Omega_{v, w_{0}}$, except that $\Omega_{v, w}-1$ is a possible exception for $w \in\{v-5, v-6, v-7\}$.

Any possible exceptions occur in the range between $\Omega_{v, v-5}-\Delta_{v, v-5}$ and $\Omega_{v, v-8}$. To deal with these possible exceptions, we repeat the calculation above but with a tighter condition, for $m \in\{v-6, v-7, v-8\}$. For $w \geq v-7$, we have $\Delta_{v, w} \geq 2$ (using $v \geq 12$ ). Thus for these values of $w$ Theorem 3.1 still gives one or more consecutive sizes, from $\Omega_{v, w}-\Delta_{v, w}$ to $\Omega_{v, w}-2$. Restricting our attention to this range, the smallest cardinality with $w=m$ (namely $\Omega_{v, m}-\Delta_{v, m}$ ) is at most one more than the largest cardinality with $w=m+1$ (namely $\left.\Omega_{v, m+1}-2\right)$ if $-\frac{m(2 v-9-m)}{2}-\left\lfloor\frac{v+2 m-8}{3}\right\rfloor \leq-\frac{(m+1)(2 v-9-(m+1))}{2}-1$; that is, $m \geq\left\lceil\frac{2 v-4}{5}\right\rceil$. This condition holds for all $v \geq 12$ and $m \geq v-8$. Thus we have a $d_{m}(v)$ of every size between $\Omega_{v, v-5}-\Delta_{v, v-5}$ and $\Omega_{v, v-8}$, which implies that we have dealt with the possible exceptions, and so in fact we have a $d_{m}(v)$ of every size between $\Omega_{v, v-5}-\Delta_{v, v-5}$ and $\Omega_{v, w_{0}}$. The result follows by Theorem 3.1 and the evaluation of $\Omega_{v, w_{0}}$.

\section{Concluding Remarks}

The largest construction we know for a general $v$ is Construction 2 with $w=0$ and $k=1$; it has size $\left(v^{3}-3 v^{2}-16 v+30\right) / 6$. By Theorem 3.1 (with $w=0$ ), we can in fact construct 
a $d_{m}(v)$ of each of the $\left\lfloor\frac{v-2}{3}\right\rfloor$ consecutive sizes up to this value. At the opposite end of the spectrum, Theorem 3.1 with $w=v-5$ gives $v-7$ consecutive sizes, with the smallest being $\left(v^{3}-6 v^{2}+5 v^{2}+6\right) / 6$. This is more or less the result given in [2]. But Theorem 3.2 significantly improves this result when $v \geq 12$; asymptotically, [2] gives approximately $v$ consecutive sizes, but Theorem 3.2 shows that Constructions 2 and 3 give approximately $9 v^{2} / 50$ consecutive sizes. This is (asymptotically) over one third of the range between the smallest and largest minimal defining sets known.

\section{References}

[1] S. Akbari, H.R. Maimani and Ch. Maysoori, Minimal defining sets for full $2-(v, 3, v-2)$ designs, Australas. J. Combin. 23 (2001), 5-8.

[2] D. Donovan, J. Lefevre, M. Waterhouse and E. Ş. Yazıcı, On defining sets of full designs with block size three, Graphs Combin. 25 (2009), 825-839.

[3] R. A. H. Gower, Minimal defining sets in a family of Steiner triple systems, Australas. J. Combin. 8 (1993), 55-73.

[4] B. D. Gray, R. Mathon, T. Moran and A. P. Street, The spectrum of minimal defining sets of some Steiner systems, Discrete Math. 261 (2003), 277-284.

[5] K. Gray, On the minimum number of blocks defining a design, Bull. Austral. Math. Society 41 (1990), 97-112.

[6] K. Gray, Further results on smallest defining set of well known designs, Australas. J. Combin. 1 (1990), 91-100.

[7] K. Gray, Defining sets of single-transposition-free designs, Util. Math. 38 (1990), 97103.

[8] K. Gray and A. P. Street, On defining sets of full designs and of designs related to them, J. Combin. Math. Combin. Comput. 60 (2007), 97-104.

[9] K. Gray and A. P. Street, Constructing defining sets of full designs, Util. Math. 76 (2008), 91-99.

[10] K. Gray and A. P. Street, Defining Sets, Section IV.13 in: CRC Handbook of Combinatorial Designs, 2nd ed. (eds. C. J. Colbourn and J. H. Dinitz), CRC Press, Boca Raton, 2007, pp. 382-385. 
[11] G. Havas, J. L. Lawrence, C. Ramsay, A. P. Street and E. Ş. Yazıcı, Defining set spectra for designs can have arbitrarily large gaps, Util. Math. 75 (2008), 67-81.

[12] E. Kolotoğlu, A new algorithm for finding the complete list of minimal defining sets of t-designs, Master Thesis, Koç University, July 2007.

[13] E. Kolotoğlu and E. Ş. Yazıcı, On minimal defining sets of full designs and selfcomplementary designs, and a new algorithm for finding defining sets of t-designs, Graphs Combin. 26 (2010), 259-281. 